\title{
Positive decomposition of transfer functions with multiple poles
}

\author{
Béla Nagy ${ }^{1}$, Máté Matolcsi² ${ }^{2}$ and Márta Szilvási
}

1 Department of Analysis, Technical University of Budapest (BME), H-1111, Budapest, Egry J. u. 1., bnagy@math.bme.hu

2 Rényi Institute of Mathematics, H-1053, Budapest, Reáltanoda u. 13-15 matomate@renyi.hu

3 Department of Analysis, Technical University of Budapest (BME), H-1111, Budapest, Egry J. u. 1., szivasi@math.bme.hu

Summary. We present new results on decomposing the transfer function $t(z)$ of a linear, asymptotically stable, discrete-time SISO system as a difference $t(z)=$ $t_{1}(z)-t_{2}(z)$ of two positive linear systems. We extend the results of [4] to a class of transfer functions $t(z)$ with multiple poles. One of the appearing positive systems is always 1-dimensional, while the other has dimension corresponding to the location and order of the poles of $t(z)$. Recently, in [10], a universal approach was found, providing a decomposition for any asymptotically stable $t(z)$. Our approach here gives lower dimensions than [10] in certain cases but, unfortunately, at present it can only be applied to a relatively small class of transfer functions, and it does not yield a general algorithm.

\section{Introduction}

Assume we are given the transfer function

$$
t(v)=\frac{p_{1} v^{n-1}+\ldots+p_{n}}{v^{n}+q_{1} v^{n-1}+\ldots+q_{n}} ; p_{j}, q_{j} \in \mathbb{R} \text { for all } 1 \leq j \leq n
$$

of a discrete time-invariant linear SISO system of McMillan degree $n$. As a normalization we also assume, as in [4], that $t(v)$ is asymptotically stable, i.e. its poles lie in the open unit disk.

The original positive realization problem is to find, if possible, a triple $A \in$ $\mathbb{R}^{N \times N}, b, c \in \mathbb{R}^{N}$ with nonnegative entries, such that $t(v)=c^{T}(v I-A)^{-1} b$ holds. The minimality problem is to find the minimal possible value of $N$. (Clearly, $N \geq n$.) The nonnegativity condition is a consequence of underlying physical constraints in some applications such as the design of charge routing networks (CRN's). Due to the nonnegativity constraint positive filters are heavily restricted in their achieveable performance. However, as pointed out in 
the seminal paper [4], one can try to decompose an arbtitrary transfer function as the difference of two positive systems and thus remove the performance limitations and retain the advantages offered by CRN's at the same time. Therefore, we are interested in decopositions of the form $t(v)=t_{1}(v)-t_{2}(v)$, where $t_{1}(v)$ and $t_{2}(v)$ are transfer functions with positive realizations of dimension $N_{1}$ and $N_{2}$. Preferably, one would like to have an a priori upper bound on the numbers $N_{1}, N_{2}$, in terms of the location and order of the poles of $t(v)$. This problem, which we will refer to as the positive decomposition problem, was solved in [4] for a wide range of transfer functions $t(v)$. Indeed, under the assumption that $t(v)$ has exclusively simple (but possibly complex!) poles, it was shown that one can take $N_{2}=1$, and a good upper bound on the value of $N_{1}$ was presented (see Theorem 8 in [4]). In particular, the 1-dimensional system was taken as $t_{2}(v)=\frac{R}{v-w}$, where $R>0$ and $0<w<1$ are appropriately chosen parameters. Then $t_{1}(v)=t(v)+t_{2}(v)$ was shown to admit a positive realization of some dimension $N_{1}$ depending on the location and the number of poles of $t(v)$. The case of transfer functions with multiple poles was left open (see the Concluding Remarks of [4]). A slight improvement on the value of $N_{1}$ was given in [8], where nonnegative simple poles with negative residues were handled in a more efficient way. Later in [9], the open case of nonnegative multiple poles was settled. Recently, in [10], a universal algorithm was found, providing a solution to the positive decomposition problem for any asymptotically stable transfer function $t(v)$ (see Theorem 4 in [10]). In fact, keeping $t_{2}(v)$ 1-dimensional, a unified, easy-to-compute method was given to find a positive system $t_{1}(v)$, while keeping good control of the dimension $N_{1}$. In most cases, however, minimality of the dimension $N_{1}$ could not be claimed (nor was it claimed in [4]). In this paper we consider a special class of transfer functions with complex multiple poles for which the dimension $N_{1}$ given in [10] can be improved. Our approach here, however, does not cover all transfer functions. In fact, the class of transfer functions considered in this note is quite narrow, so that we regard the main contribution here not as much in the achieved results but rather in the essentially new ideas behind the construction of Theorem 2 below. With further research in the future these ideas may well lead to a general improvement of the algorithm of [10].

For the general theory and applications of positive linear systems we refer the interested reader to [5]. Also, a thorough overview of recent results related to the positive realization problem has been given in [2]. For direct applications of the positive decomposition problem see [4] and [3].

\section{Decomposition of transfer functions}

As described in the Introdction, we are looking for positive systems $t_{1}(v)$ and $t_{2}(v)$ such that $t_{2}(v)$ is 1-dimensional and the decomposition $t(v)=t_{1}(v)-$ $t_{2}(v)$ holds. Thus, we take $t_{2}(v)=\frac{p}{v-w}$ with some choice of $0<p, 0<w<1$, and we are looking for a positive realization of the trasfer function $t_{1}(v)$, as 
in [4]. Therefore, we are basically led back to the original positive realization problem for $t_{1}(v)$. In the construction of a positive realization of $t_{1}(v)$ it is customary to make use of the following well-known result from positive system theory (for a proof see e.g. [1]). We also remark that this 'geometric' lemma is utilized in most constructions concerning positive realizations (see e.g. [1, $4,10])$.

Lemma 1. Let $t(v)$ be a rational transfer function as in (1), and let $(c, J, b)$ be any minimal realization of $t(v)$, i.e. $t(v)=c(v I-J)^{-1} b$, and the dimensions of the matrices $(c, J, b)$ are $1 \times n, n \times n, n \times 1$, respectively. Assume that there exists a system invariant polyhedral cone $\mathcal{P} \subset \mathbb{R}^{n}$, i.e. a finitely generated cone $\mathcal{P} \subset \mathbb{R}^{n}$ such that $b \in \mathcal{P}, J \mathcal{P} \subset \mathcal{P}$ and $c \cdot p \geq 0$ for all $p \in \mathcal{P}$. If the number of extremal rays of $\mathcal{P}$ is $N$ then there exists a positive realization of $t(z)$ of dimension $N$.

We will first review here the results of [10] concerning the case of transfer functions with negative real multiple poles. This case gives good indication as to what dimension $N_{1}$ we should expect for transfer functions with multiple poles in general (see Remark 2 below). We will also see that here we can even claim minimality of $N_{1}$ in certain cases (see Remark 1 below). Besides interest of its own, we will use this result in the proof of Theorem 2 below.

Theorem 1. Let $h$ be a negative number such that $|h|<1$. If the transfer function $t(v)$ with real coefficients has exclusively the pole $h$ of order $k$, i.e.

$$
t(v)=\sum_{j=1}^{k} \frac{\alpha_{j}}{(v-h)^{j}},
$$

then there are positive numbers $p$ and $w$ such that $|h|<w<1$, and the function

$$
t_{1}(v):=t(v)+\frac{p}{v-w}
$$

has a nonnegative realization of dimension $N_{1} \leq 2 k$.

Proof. We only include the idea of the proof here (see [10] for details).

Consider a real Jordan minimal realization $(c, A, b)$ of $t(v)$ (where $A$ is a modified Jordan block, cf. [10]).

Let $|h|<w<1$, and $b_{0}, c_{0}>0$ sufficiently large, and define $p=b_{0} c_{0}$, $t_{1}(v)=t(v)+\frac{p}{v-w}$. Also, let $I_{1}$ denote the $1 \times 1$ identity matrix. Then the triple

$$
\left[\left(c_{0} c\right), w I_{1} \oplus A,\left(\begin{array}{c}
b_{0} \\
b
\end{array}\right)\right]
$$

is a minimal Jordan ralization of $t_{1}(v)$.

Consider the following vectors $u_{j} \in \mathbb{R}^{k+1}(j=1,2, \ldots, 2 k)$, (where ${ }^{T}$ denotes transpose): 
$u_{1}:=(1,1,0,0, \ldots, 0)^{T}, u_{2}:=(1,-1,0,0, \ldots, 0)^{T}, u_{3}:=(1,0,1,0, \ldots, 0)^{T}$, $u_{4}:=(1,0,-1,0, \ldots, 0)^{T} \ldots ., u_{2 k}:=(1,0,0,0, \ldots,-1)^{T}$.

Consider now the polyhedral cone $\mathcal{K}_{u}$ generated by these vectors:

$$
\mathcal{K}_{u}:=\text { cone }\left[u_{1}, u_{2}, \ldots, u_{2 k}\right] .
$$

It can be shown that with a sufficiently large choice of $b_{0}, c_{0}$ the cone $\mathcal{K}_{u}$ will be a system invariant cone with respect to $\left[\left(c_{0}, c\right), w I_{1} \oplus A,\left(b_{0}, b\right)^{T}\right]$.

The number of the extreme rays of the polyhedral cone $\mathcal{K}_{u}$ is clearly $2 k$. Therefore the application of Lemma 1 gives the desired result.

Remark 1 . We can claim minimality of the dimension $N_{1}=2 k$ in certain cases. Namely, assume that the location and the order of the negative pole of $t(v)$ satisfy the condition $|h| k \geq k-1$. Then, any positive realization $(c, A, b)$ of the function $t_{1}(v)$ must be of dimension at least $N_{1} \geq 2 k$, due to the following argument (in which we combine ideas from [1] and [7]). The dominant pole of $t_{1}(z)$ is $w$, therefore we can assume without loss of generality that all eigenvalues $\lambda$ of $A$ satisfy $|\lambda| \leq w$ (see Theorem 3.2 in [1]). Also, the trace of $A$ is clearly nonnegative. Combining these facts we get $0 \leq \operatorname{Tr} A \leq h k+$ $\left(N_{1}-k\right) w$, which implies $\left(N_{1}-k\right) w \geq-h k \geq k-1$, hence $N_{1} \geq 2 k$.

On the one hand, this argument shows that, in general, the appearence of the pole order $k$ as a factor in the dimension $N_{1}$ should not be unexpected. On the other hand, it also shows that an attempt to improve the result of Theorem 1 must take into account the value $h k$ in some way.

Remark 2. The result of Theorem 1 also gives an indicaton as to what dimension $N_{1}$ to expect in the presence of complex multiple poles. In [4], in the case of one pair of conjugate complex simple poles $z$ and $\bar{z}$, the dimension of a positive realization of $t_{1}(v)$ was given as the smallest integer $m$ such that $z$ lies in the interior of the regular polygon $\mathcal{P}_{m}$ with $m$ edges inscribed in the unit circle and having one vertex at 1 (see Proposition 7 in [4]). Then, in the spirit of Theorem 1 above, one could expect that if $z$ and $\bar{z}$ are poles of order $k$, then $t_{1}(v)$ admits a positive realization of dimension $N_{1}=k m$ with $m$ as above. However, the general method of [10] gives a slightly worse result (cf. Theorem 1 in [10]), especially for poles located near the vertices of $\mathcal{P}_{m}$, i.e. close to the boundary of the unit disk. In fact, Theorem 1 in [10] gives $N_{1} \leq 4 k(Q+1)$, where $Q \approx\left[\frac{\log \sqrt{2}}{-\log \mid z]}\right]$, where [.] denotes the integer part of a number.

Now we turn to transfer functions with complex multiple poles. We remark that the unified algorithm given in Theorem 4 of [10] covers all such transfer functons, too. However, the dimension $N_{1}$ given in [10] is, in general, not minimal, and can be improved in certain cases. We will treat such a case below. Our approach here, however, concerns a special class of transfer functions only, and it requires further research in the future to extend it to a universal algorithm. The essentially new feature of the construction below is that it is not based on the geometric Lemma 1, but rather on some simple algebraic manipulations. 
Theorem 2. Assume that $z=a+b \mathrm{i}$ and $\bar{z}=a-b \mathrm{i}$ are located in the right hand half of the interior of the square $\mathcal{P}_{4}$, i.e. $a \geq 0$ and $a+|b|<1$, and that the transfer function $t(v)$ is of the form

$$
t(v)=\frac{\alpha}{(v-z)^{k}(v-\bar{z})^{k}} \quad \alpha \in \mathbb{R} .
$$

Then there are positive numbers $p$ and $w$ such that $|z|<w<1$, and the function

$$
t_{1}(v):=t(v)+\frac{p}{v-w}
$$

has a nonnegative realization of dimension $N_{1} \leq 1+7 k$.

Proof. The proof is based on some simple algebraic manipulations.

Observation 1. If $a \geq 0$ and $t(v)$ has a positive realization $(c, A, b)$, then $t(v-a)$ has a positive realization $(c, A+a I, b)$ of the same dimension.

Observation 2. If $t(v)$ has a positive realization of dimension $N$ then $v^{j} t\left(v^{p}\right)$ (where $0 \leq j \leq p-1$ ) has a positive realization of dimension $p N$ (for a constructive proof see $[6])$.

Observation 3. Assume a transfer function $t(v)$ has impulse response sequence $\left(h_{1}, h_{2}, \ldots\right)$, i.e. $t(v)=\sum_{j=1}^{\infty} h_{j} v^{-j}$, and $t(v)$ has a positive realization $\left(c^{T}, A, b\right)$ of dimension $N$. Then the function $t^{(2)}(v)$ corresponding to the sequence $\left(0, h_{1}, 2 h_{2}, 3 h_{3}, \ldots\right)$, i.e. $t^{(2)}(v)=\sum_{j=1}^{\infty} j h_{j} v^{-j-1}$, has a positive realization $c_{2}^{T}:=\left(c^{T}, 0^{T}\right), A_{2}:=\left(\begin{array}{ll}A & I \\ 0 & A\end{array}\right)$ and $b_{2}:=\left(\begin{array}{l}0 \\ b\end{array}\right)$ of dimension $2 N$. More generally, for any value $m \geq 1$ the transfer function $t^{(m)}(v)$ corresponding to the sequence $\left(0,0, \ldots, 0, h_{1},\left(\begin{array}{c}m \\ m-1\end{array}\right) h_{2},\left(\begin{array}{c}m+1 \\ m-1\end{array}\right) h_{3},\left(\begin{array}{c}m+2 \\ m-1\end{array}\right) h_{4}, \ldots\right)$, where the number of the initial $0 \mathrm{~s}$ is $m-1$, has a positive realization of dimension $m N$ as $c_{m}^{T}:=\left(c^{T}, 0^{T}, \ldots, 0^{T}\right)$ and

$$
b_{m}:=\left(\begin{array}{c}
0 \\
0 \\
\vdots \\
b
\end{array}\right) \text { and } A_{m}:=\left(\begin{array}{ccccc}
A & I & 0 & \ldots & 0 \\
0 & A & I & \ldots & 0 \\
\ldots & \ldots & \ldots & \ldots & \ldots \\
\ldots & \ldots & \ldots & A & I \\
0 & 0 & 0 & \ldots & A
\end{array}\right)
$$

This is straightforward to check.

With these observations at hand we are ready to prove our Theorem. Write

$$
\begin{array}{r}
t_{1}(v)=\left[\frac{p}{v-w}-\frac{|\alpha|}{\left((v-a)^{2}-b^{2}\right)^{k}}\right]+\left[\frac{\alpha}{\left((v-a)^{2}+b^{2}\right)^{k}}+\frac{|\alpha|}{\left((z-a)^{2}-b^{2}\right)^{k}}\right]= \\
=: f_{1}(v)+f_{2}(v),
\end{array}
$$

where $f_{1}$ and $f_{2}$ denote the functions in the brackets.

Notice that $f_{1}(v)$ has real poles $a+b$ and $a-b$, both of order $k$. Therefore, 


$$
\begin{array}{r}
f_{1}(v)=\left[\frac{p / 2}{v-w}+\sum_{j=1}^{k} \frac{c_{j}}{(v-a-b)^{j}}\right]+\left[\frac{p / 2}{v-w}+\sum_{j=1}^{k} \frac{c_{j}}{(v-a+b)^{j}}\right]= \\
g_{1}(v)+g_{2}(v) .
\end{array}
$$

Note also that at least one of the numbers $a+b, a-b$, say $a+b$, is nonnegative. If we choose $a+|b|<w<1$, and $p$ sufficiently large, then we can apply Corollary 1 of [9] in order to obtain a positive realization of $g_{1}(v)$ of dimension $k+1$ (notice that besides the dominant pole $w, g_{1}(v)$ has only the nonnegative multiple pole $a+b$, therefore Corollary 1 of [9] can indeed be applied). If, in addition, $a-b$ is also nonnegative then the same applies to $g_{2}(v)$, giving a positive ralization of $g_{2}(v)$ of dimension $k+1$. In the worse case of $a-b$ being negative, Theorem 1 above can be applied to obtain a positive realization of $g_{2}(v)$ of dimension $2 k$. Then we can consider the parallel connection of the positive systems of $g_{1}(v)$ and $g_{2}(v)$ in order to obtain a positive realization of $f_{1}(v)=g_{1}(v)+g_{2}(v)$. Therefore, in any case, the function $f_{1}(v)$ admits a positive ralization of dimension not more than $3 k+1$.

Now, we find a nonnegative realization of $f_{2}(v)$ in the following way. First, we find a positive realization of dimension 2 of the function

$$
h(v)=\frac{\alpha}{v+b^{2}}+\frac{|\alpha|}{v-b^{2}}
$$

(note that $h(v)$ is a 2 -dimensional system with nonegative impulse response, and a simple construction for positive realization of such systems is included e.g. in Lemma 3 of [7]). Then we use Observation 3 with $m=k$. A simple calculation shows that (with notation as in Observation 3)

$$
h^{(k)}(v)=\frac{\alpha}{\left(v+b^{2}\right)^{k}}+\frac{|\alpha|}{\left(v-b^{2}\right)^{k}} .
$$

Therefore the construction of Observation 3 yields a positive realization of $h^{(k)}(v)$ of dimension $2 k$. Then we apply Observation 2 with $j=0, p=2$ in order to obtain a positive realization of

$$
h^{(k)}\left(v^{2}\right)=\frac{\alpha}{\left(v^{2}+b^{2}\right)^{k}}+\frac{|\alpha|}{\left(v^{2}-b^{2}\right)^{k}}
$$

of dimension $4 k$. Finally, we use Observation 1 to obtain a positive realization of

$$
f_{2}(v)=h^{(k)}\left((v-a)^{2}\right)=\frac{\alpha}{\left((v-a)^{2}+b^{2}\right)^{k}}+\frac{|\alpha|}{\left((v-a)^{2}-b^{2}\right)^{k}},
$$

also in $4 k$ dimensions.

The paprallel connection of the positive realizations of $f_{1}(v)$ and $f_{2}(v)$ gives a positive realization of $t_{1}(v)$ of dimension $1+7 k$. 
Remark 3. At this moment, the obvious disadvatage of the method presented above is that it applies only to a small class of transfer functions $t(v)$. The location of the poles is restricted to the right hand half of $\mathcal{P}_{4}$, and also $t(v)$ must contain only one fraction term. However, there may be hope that with a more effective use of Observatons 1, 2, 3 above (or, with the addition of further observations) one can produce a general solution of the positive decomposition problem, such as the one described in Theorem 4 of [10], but maybe with a smaller dimension $N_{1}$.

\subsection{An example}

As an illustration we give a specific example where Theorem 2 above gives a better result than the general algorithm of [10]. Consider the transfer function

$$
t(v)=\frac{1}{\left(v^{2}-0.4 v+0.53\right)^{2}} .
$$

Then, $t(v)$ has the poles $z=0.2+0.7 \mathrm{i}$ and $\bar{z}=0.2-0.7 \mathrm{i}$, both of order 2 . Also, $|z| \approx 0.728$, and we have $Q=1$ in Theorem 1 of [10]. Therefore, the construction of Theorem 1 of [10] gives a positive realization of

$$
t_{1}(v)=\frac{p}{v-w}+\frac{1}{\left(v^{2}-0.4 v+0.53\right)^{2}}
$$

of dimension 16 (with appropriately chosen parameters $p, w$ ), while Theorem 2 above gives a positive realization of dimension 15 .

\section{Conclusion}

In this paper we considered decompositions $t(v)=t_{1}(v)-t_{2}(v)$ of a transfer function $t(v)$ as a difference of two positive systems $t_{1}(v)$ and $t_{2}(v)$. We keep $t_{2}(v)$ 1-dimensional while keeping good control of the dimension of $t_{1}(v)$. Such decompositions are important due to the inherent positivity of certain networks in applications, such as CRN's. Recently, a unified and universal solution to the positive decomposition problem for any transfer function $t(v)$ was provided in [10]. However, minimality of the dimension of the positive realization of $t_{1}(v)$ is not claimed in [10], and in this note we considered a class of transfer functions for which the dimension can be lowered. Our approach here contains essentially new ideas and is based on simple algebraic observations. While the class of transfer functions considered in this note is fairly narrow, there may be hope to extend the results to a general algorithm in the future. 


\section{References}

1. B.D.O. Anderson, M. Deistler, L. Farina, and L. Benvenuti, "Nonnegative realization of a linear system with nonnegative impulse response," IEEE Trans. Circuits Syst. I, vol. 43, no.2, pp 134-142, Feb. 1996.

2. L. Benvenuti and L. Farina, "A tutorial on the positive realization problem", IEEE Trans. Automat. Control vol. 49, no. 5, pp. 651-664, 2004.

3. L. Benvenuti and L. Farina, "The design of fiber-optic filters", Journal of Lightwave Technology, vol. 19, pp. 1366-1375, 2001.

4. L. Benvenuti, L. Farina, and B.D.O. Anderson, "Filtering through a combination of positive filters," IEEE Trans. Circuits Syst. I, vol. 46, pp. 1431-1440, Dec. 1999.

5. L. Farina and S. Rinaldi, Positive linear systems: Theory and applications. New York: Wiley, 2000.

6. K.-H. Foerster and B. Nagy, "Nonnegative realizations of matrix transfer functions", Lin. Algebra Appl., vol. 311, pp. 107-129, 2000.

7. C. Hadjicostis, "Bounds on the size of minimal nonnegative realizations for discrete-time LTI systems," Syst. Contr. Lett., vol. 37, pp 39-43, 1999.

8. A. Halmschlager and M. Matolcsi, "Minimal positive realizations for a class of transfer functions", IEEE Trans. Circuits Syst. II, to appear.

9. B. Nagy, M. Matolcsi, "Minimal Positive Realizations of Transfer Functions with Nonnegative Multiple Poles," IEEE Trans. Automat. Control vol. 50, no. 9, pp. 1447-1450, September, 2004.

10. B. Nagy, M. Matolcsi and Márta Szilvási, "Order Bound for the Realization of a Combination of Positive Filters", preprint submitted to IEEE Tran. Aut. Cont. (electronic version available at

http://www.math.bme.hu/ matolcsi/electronicpapers.htm). 